\title{
Influencia de los diferentes tamaños granulométricos en la superficie específica Blaine y resistencias mecánicas del cemento portland
}

\author{
JULIAN REZOLA IZAGUIRRE \\ Ingeniero Químico y Director del Laboratorio \\ Central de Cementos Rezola, S. A.
}

Recientemente observamos una marcada tendencia, y verdadero interés, de obtener un mejor conocimiento de la influencia y comportamiento de los tamaños granulométricos del cemento portland. Prueba de ello es que son yà varias las publicaciones que se han ocupado de estos pormenores, dándose el caso paradójico de que mucho antes de contar con los medios instrumentales actuales, ya han existido autores que se han anticipado en emitir su opinión sobre el particular.

Como se comprenderá después de esto, es lógico que por nuestra parte fuéramos un tanto escépticos a la hora de admitir las conclusiones establecidas en estas condiciones. En vista de ello, no hemos vacilado un instante, disponiendo de un separador centrífugo, para iniciar una investigación acerca del particular, tratando de eliminar estos inconvenientes.

Como es lógico suponer, en este caso, las conclusiones que se establezcan, como las directrices que emanen de las mismas, serán fruto de un estudio totalmente experimental.

En un estudio previo se estimó conveniente el que los ensayos se realizaran con clínker sólo, por aquello de ir eliminando variantes, ya que de lo contrario y sobre todo si lo realizábamos con cemento bien binario o ternario, la interacción y comportamiento de los diferentes materiales nos iban a afectar sensiblemente.

En vista de ello y debido al criterio establecido, realizamos una molienda de clínker sólo. Los resultados obtenidos han sido los siguientes:

\begin{tabular}{|c|c|c|c|}
\hline \multirow{2}{*}{ Tamaños $\mu$} & \multirow{2}{*}{ Inferiores } & \multicolumn{3}{|c|}{ R E S I D U O S } \\
\cline { 2 - 2 } & & \% Parciales & $\%$ Acumulados \\
\cline { 1 - 2 } 55 & 68,7 & 31,3 & 31,3 \\
48 & 65,7 & 3,0 & 34,3 \\
39 & 60,6 & 5,1 & 39,4 \\
22,5 & 47,3 & 12,7 & 52,1 \\
14,5 & 32,9 & 15,0 & 67,1 \\
9,9 & 18,9 & 14,0 & 81,1 \\
7,1 & 9,2 & 9,7 & 90,8 \\
3,7 & 3,7 & 5,5 & 96,3 \\
& & 3,7 & 100,0 \\
\hline
\end{tabular}


Posteriormente, al clínker molido se le ha sometido, en el Elutriator BHACO, a separaciones sucesivas; por eliminación de las partículas menores y de esta forma se prepararon las muestras pertinentes.

En cada una de las muestras se ha determinado, primero, la superficie específica Blaine, siendo los resultados obtenidos los que figuran en el cuadro 1.

C U A D R O 1

Influencia del tamaño granulométrico en la superficie Blaine

\begin{tabular}{|c|c|c|c|c|c|c|}
\hline & & \multirow{2}{*}{$\begin{array}{c}\text { Cemento } \\
\text { inicial }\end{array}$} & \multicolumn{4}{|c|}{ Tamaño granulométrico eliminado } \\
\hline & & & $<3,7 \mu$ & $<7,1 \mu$ & $<9,9 \mu$ & $<14,5 \mu$ \\
\hline \multicolumn{2}{|l|}{ Superficie Blaine $\left(\mathrm{cm}^{2} / \mathrm{g}\right)$} & 3,139 & 2.697 & 2.665 & 2.050 & 1.333 \\
\hline \multirow{2}{*}{ Diferencias en Blaine: } & parciales & 0 & -442 & -32 & - 615 & -717 \\
\hline & acumulados & 0 & -442 & -474 & -1.089 & -1.806 \\
\hline \multirow{2}{*}{$\%$ Tamaños eliminados: } & parciales & 0 & 3,7 & 5,5 & 9,7 & 14 \\
\hline & acumulados & 0 & 3,7 & 9,2 & 18,9 & 32,9 \\
\hline \multirow{2}{*}{$\begin{array}{l}\text { Influencia de la super- } \\
\text { ficie Blaine por cada } 1 \% \\
\text { de tamaño eliminado: }\end{array}$} & parcial & 0 & $-119,4$ & $-5,8$ & $\rightarrow 61,3$ & $-\quad 51,2$ \\
\hline & acumulado & 0 & $-119,4$ & $-52,6$ & $-\quad 57,6$ & $-\quad 54,9$ \\
\hline \multirow{2}{*}{$\begin{array}{l}\text { Disminución de la super- } \\
\text { ficie Blaine expresada } \\
(\%) \text { : }\end{array}$} & acumulado & 0 & $-14,0$ & $-1,0$ & $-\quad 19,58$ & 22,8 \\
\hline & parcial & 0 & $-14,0$ & $-15,0$ & - $\quad 34,6$ & 57,5 \\
\hline
\end{tabular}

Como puede observarse, los resultados son harto elocuentes. En honor a la verdad, disponíamos ya anteriormente de referencias acerca de la influencia que ejercen los tamaños pequeños; pero es justo reconocer, por nuestra parte, que en ningún momento esperábamos que influyeran tanto, ya que el valor de $119,4 \mathrm{~cm}^{2} / \mathrm{g}$ por cada $1 \%$ de tamaños inferiores a 3,7 eliminados es digno de toda consideración.

Esto, una vez más, pone en evidencia la validez y representatividad de la superficie Blaine.

Aun cuando en el cuadro 1 figuran sólo los resultados obtenidos con la eliminación de $14,5 \mu$, las separaciones realizadas han alcanzado las 22,5 ; pero, debido a que con esta muestra la determinación de la superficie Blaine no nos inspira demasiada confianza, hemos preferido no incluirlos.

Anteriormente hemos indicado que las muestras son de clínker sólo; por lo tanto, ya que entramos ahora a tratar pormenores de las resistencias mecánicas, nos hemos visto obligados a mezclar a las respectivas muestras una cantidad constante de yeso previamente molido. 
La adopción de esta medida obedece a que la composición química de las diferentes muestras no presentan diferencias marcadas.

Prueba de ello son los resultados que figuran en el cuadro 2.

CUADRO 2

\begin{tabular}{|c|c|c|c|c|c|c|c|}
\hline \multirow{2}{*}{$\begin{array}{c}\text { Composición } \\
\text { química }\end{array}$} & \multirow{2}{*}{$\begin{array}{c}\text { Cemento } \\
\text { inicial }\end{array}$} & \multicolumn{6}{|c|}{ Tamaño granulométrico eliminado } \\
\hline & & $<3,7 \mu$ & $<7,1 \mu$ & $<9,9 \mu$ & $<14,5 \mu$ & $<22,5 \mu$ & $<39 \mu$ \\
\hline P. F. & 1,38 & 1,36 & 1,33 & 1,14 & 1,06 & 1,10 & 0,97 \\
\hline R. I. & 0,52 & 0,62 & 0,64 & 0,49 & 0,50 & 0,40 & 0,38 \\
\hline $\mathrm{SiO}_{2}$ & 20,76 & 20,68 & 20,60 & 20,65 & 20,50 & 21,04 & 20,62 \\
\hline $\mathrm{Al}_{2} \mathrm{O}_{3}$ & 7,88 & 7,56 & 7,82 & 7,48 & 7,58 & 7,48 & 7,84 \\
\hline $\mathrm{Fe}_{2} \mathrm{O}_{3}$ & 2,56 & 2,60 & 2,54 & 2,54 & 2,54 & 2,54 & 2,50 \\
\hline $\mathrm{CaO}$ & 63,80 & 63,70 & 63,70 & 64,20 & 64,30 & 63,84 & 63,65 \\
\hline $\mathrm{MgO}$ & 1,45 & 1,36 & 1,46 & 1,37 & 1,34 & 1,39 & 1,40 \\
\hline $\mathrm{SO}_{3}$ & 0,62 & 0,48 & 0,53 & 0,49 & 0,53 & 0,35 & 0,25 . \\
\hline
\end{tabular}

Fijándonos detenidamente en cada una de ellas, quizá en una primera observación nos parezca que la correspondiente a las $22,5 \mu$ tenga un contenido en sílice más alto, así mismo, si tenemos en cuenta el contenido en R. I., veremos que la suma de ambos, expresada en componentes fijos, sigue manteniendo la diferencia.

No obstante, somos de la opinión de que dicha diferencia obedece más bien a errores experimentales, ya que pretender atribuir a variaciones o posibles segregaciones del propio clínker, es decir, que la composición química de los tamaños difiera de unos a otros, nos hace pensar que el establecer esta hipótesis resulte un tanto aventurado, aun cuando hay autores que dicen que el $\mathrm{SC}_{3}$ se presenta en mayor cuantía dentro de los tamaños pequeños.

Si hubiéramos observado aunque no sea más que una simple tendencia, en alguno de los componentes, entonces sí podríamos pensar que existiera alguna diferencia, pero como en el orden práctico no vemos nada acerca del particular somos, como hemos dicho antes, un tanto escépticos en admitir que suceda tal segregación y más aún en nuestro caso concreto tratándose, como se trata, de un clínker de horno Lepol.

Una vez preparadas las muestras de clínker molido, previa separación del tamaño granulométrico correspondiente, se le ha adicionado yeso, también molido, para luego, en una mezcladora, obtener una muestra debidamente homogeneizada.

Debido a lo penoso que resulta esta preparación, entre otros motivos por la limitación de la cantidad de material que debe tomarse para realizar la separación pertinente, no hemos podido contar con mucha cantidad de muestra disponible.

Por lo tanto, debido a esta circunstancia, hemos preferido dedicar toda la preferencia a la determinación de resistencias, por considerarlas, de momento, como más interesantes, ya que las conclusiones que pueden derivarse de éstas tienen un valor más positivo y práctico que si nos dedicamos a observar su efecto en los fraguados, estabilidad, retracciones, etc.

Los ensayos han abarcado las edades de 1,3 y 28 días; la de 7 días no la hemos experi- 
mentado, entre otras cosas, por la limitación de cantidad de muestra antes aludida y porque, mayormente, la resistencia a 7 días no aporta demasiados conocimientos.

En los cuadros 3 y 4 figuran los resultados obtenidos.

$$
\text { C U A D R O } 3
$$

Influencia del tamaño granulométrico eliminado en las resistencias a 1 día

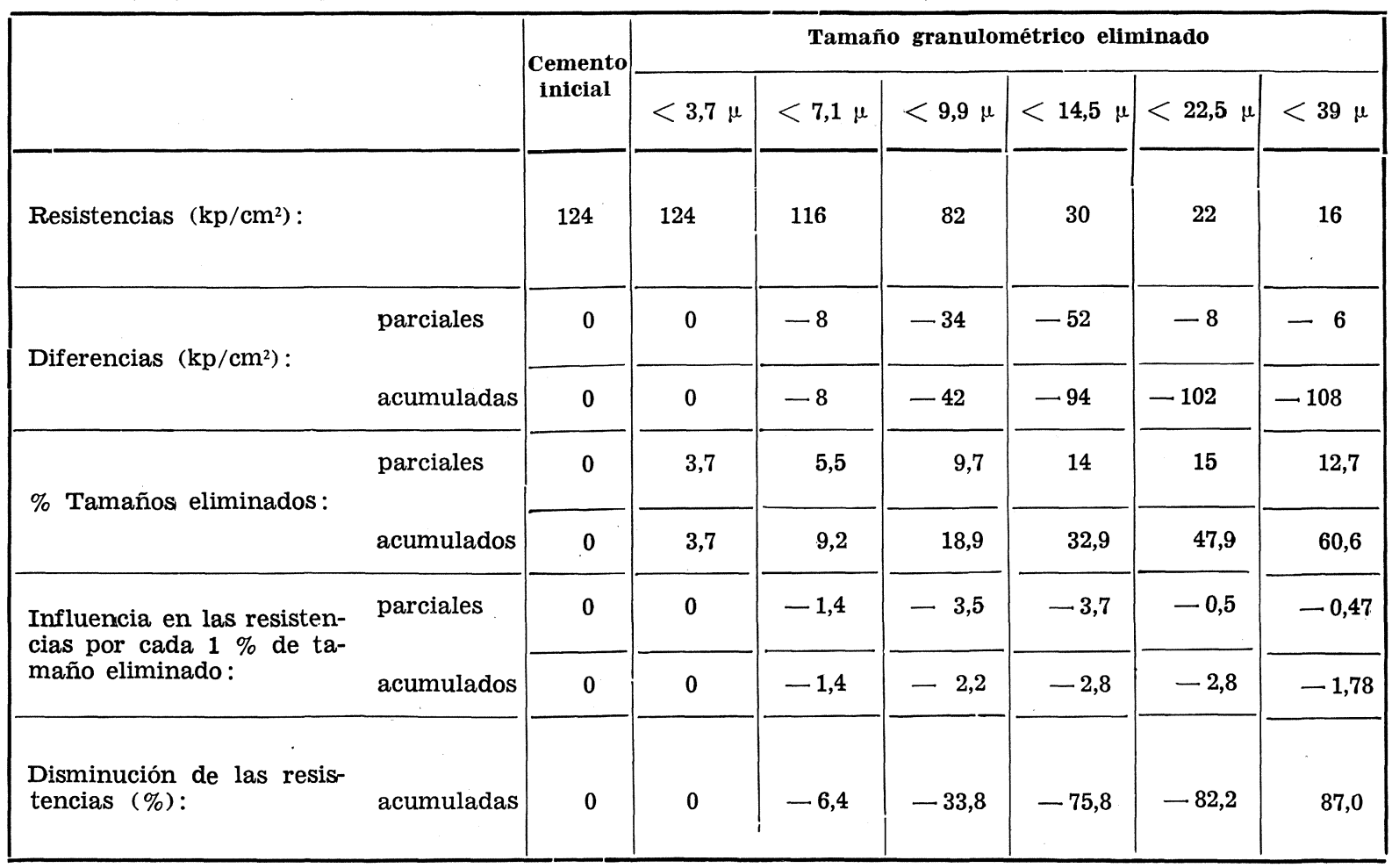

De la observación del cuadro 3 , deducimos que el efecto de la eliminación de tamaños de 3,7 micras pasa desapercibido. Con la de 7,1 se nota una ligera influencia en las resistencias, pero que a partir de ésta, es decir con la correspondiente a 9,9 micras, el efecto es ya sensible y que si se tiene en cuenta el porcentaje real de su contenido, su repercusión es patente, llegando a ser de hasta - 33,8 por 100 y ni qué decir respecto al de $39 \mathrm{mi}$ cras, que da lugar a un descenso del $87 \%$.

El que las resistencias desciendan a medida que desaparecen los tamaños finos, esto es en definitiva, cuando el cemento resulta más grueso (véase el gráfico número 1) parece lógico y más aún, si dentro del proceso de fraguado y endurecimiento del cemento existe, como parece, un proceso de hidratación junto a otros de colmatación, gelificación, cristalización, etc.

Este mismo razonamiento es aplicable a la edad de 3 días (véase el cuadro 4).

Respecto a la edad de 28 días, en cambio (véase el cuadro 5) y aún cuando en líneas generales abservamos el mismo efecto, debemos constatar que, con la eliminación de los tamaños de 14,5 micras, solamente ocasiona un descenso en las resistencias de 14,7 \%, cuando por el contrario y a la edad 1-3 días, representa una baja de 75,8 \% y 46,7 \% respectivamente. 
CURVAS GRANULOMETRICAS CORRESPONDIENTES A LAS SEPARACIONES REALIZADAS:

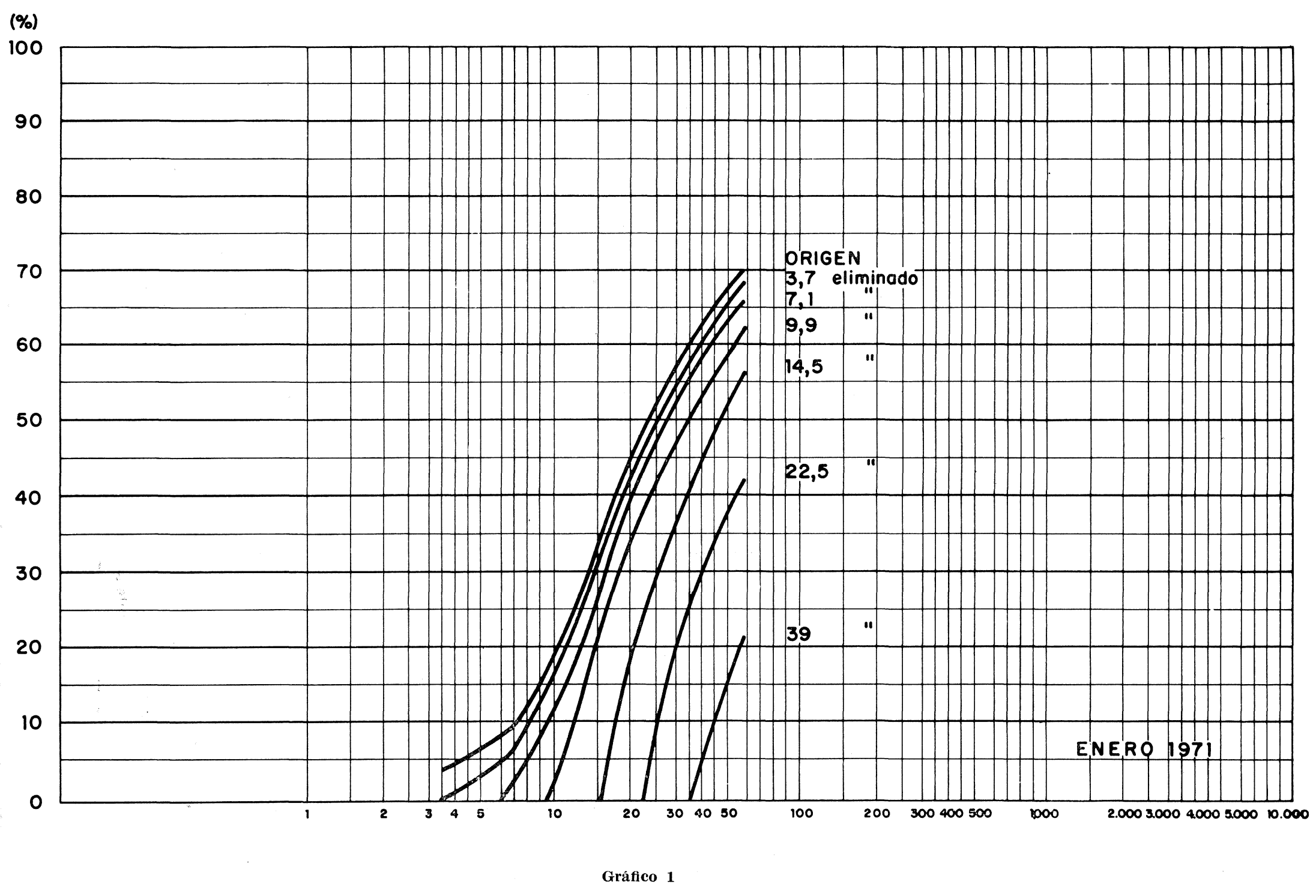


C U A D R O 4

Influencia del tamaño granulométrico eliminado en las resistencias a 3 días

\begin{tabular}{|c|c|c|c|c|c|c|c|c|}
\hline & \multirow{2}{*}{$\begin{array}{c}\text { Cemento } \\
\text { inicial }\end{array}$} & \multicolumn{6}{|c|}{ Tamaño granulométrico eliminado } \\
\hline & & & $<3,7 \mu$ & $<7,1 \mu$ & $<9,9 \mu$ & $<14,5 \mu$ & $<22,5 \mu$ & $<39 \mu$ \\
\hline \multicolumn{2}{|l|}{ Resistencias $\left(\mathrm{kp} / \mathrm{cm}^{2}\right)$ : } & 263 & 256 & 254 & 216 & 140 & 56 & 37 \\
\hline \multirow{2}{*}{ Diferencias $\left(\mathrm{kp} / \mathrm{cm}^{2}\right)$ : } & parciales & 0 & -7 & -2 & -38 & -176 & -84 & -19 \\
\hline & acumuladas & 0 & -7 & -9 & -47 & -123 & -207 & -226 \\
\hline \multirow{2}{*}{ \% Tamaños eliminados: } & parciales & 0 & 3,7 & 5,5 & 9,7 & 14 & 15 & 12,7 \\
\hline & acumulados & 0 & 3,7 & 9,2 & 18,9 & 32,9 & 47,9 & 60,6 \\
\hline \multirow{2}{*}{$\begin{array}{l}\text { Influencia en las resisten- } \\
\text { cias por cada } 1 \% \text { de ta- } \\
\text { maño eliminado: }\end{array}$} & parciales & 0 & 1,8 & 2,7 & 3,9 & 12,5 & 5,6 & 1,4 \\
\hline & acumulados & 0 & 1,8 & 0,9 & 2,4 & 8,7 & 4,3 & 3,7 \\
\hline $\begin{array}{l}\text { Disminución de resisten- } \\
\text { cias }(\%) \text { : }\end{array}$ & acumuladas & 0 & 2,6 & 3,4 & 17,8 & 46,7 & 78,7 & 85,9 \\
\hline
\end{tabular}

CUADRO 5

Influencia del tamaño granulométrico eliminado en las resistencias a 28 días

\begin{tabular}{|c|c|c|c|c|c|c|c|c|}
\hline \multirow{2}{*}{ 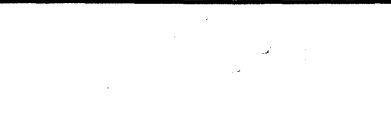 } & & \multirow{2}{*}{$\begin{array}{c}\text { Cemento } \\
\text { inicial }\end{array}$} & \multicolumn{6}{|c|}{ Tamaño granulométrico eliminado } \\
\hline & & & $<3,7 \mu$ & $<7,1 \mu$ & $<9,9 \mu$ & $<14,5 \mu$ & $<22,5 \mu$ & $<39 \mu$ \\
\hline Resistencias $\left(\mathrm{kp} / \mathrm{cm}^{2}\right)$ : & & 427 & 417 & 410 & 408 & 364 & 310 & 272 \\
\hline \multirow{2}{*}{ Diferencias $\left(\mathrm{kp} / \mathrm{cm}^{2}\right)$ : } & parciales & 0 & -11 & -6 & -2 & -44 & -54 & -38 \\
\hline & acumuladas & 0 & -11 & -17 & -19 & -63 & -117 & -155 \\
\hline \multirow{2}{*}{ \% Tamaños eliminados: } & parciales & 0 & 3,7 & 5,5 & 9,7 & 14 & 15 & 12,7 \\
\hline & acumulados & 0 & 3,7 & 9,2 & 18,9 & 32,9 & 47,9 & 60,6 \\
\hline \multirow{2}{*}{$\begin{array}{l}\text { Influencia en las resisten- } \\
\text { cias por cada } 1 \% \text { de ta- } \\
\text { maño eliminado: }\end{array}$} & parciales & 0 & 2,9 & 1,0 & 0,2 & 3,1 & 2,9 & 2,9 \\
\hline & acumuladas & 0 & 2,9 & 1,8 & 1,0 & 1,9 & 1,1 & 2,5 \\
\hline $\begin{array}{l}\text { Disminución de resisten- } \\
\text { cias }(\%) \text { : }\end{array}$ & acumuladas & 0 & 2,5 & 3,9 & 4,4 & 14,7 & 27,4 & 36,3 \\
\hline
\end{tabular}


Con esto queremos significar, y para ello nos remitimos al gráfico 2, que las resistencias referidas a 3 días descienden de una forma patente con la eliminación de determinados tamaños, así como en las de 24 horas, es decir en las edades tempranas, pero que, por el contrario, el aumento que experimenta de 3 a 28 días, es mucho más patente en los tamaños gruesos, que las que presentan los tamaños finos.

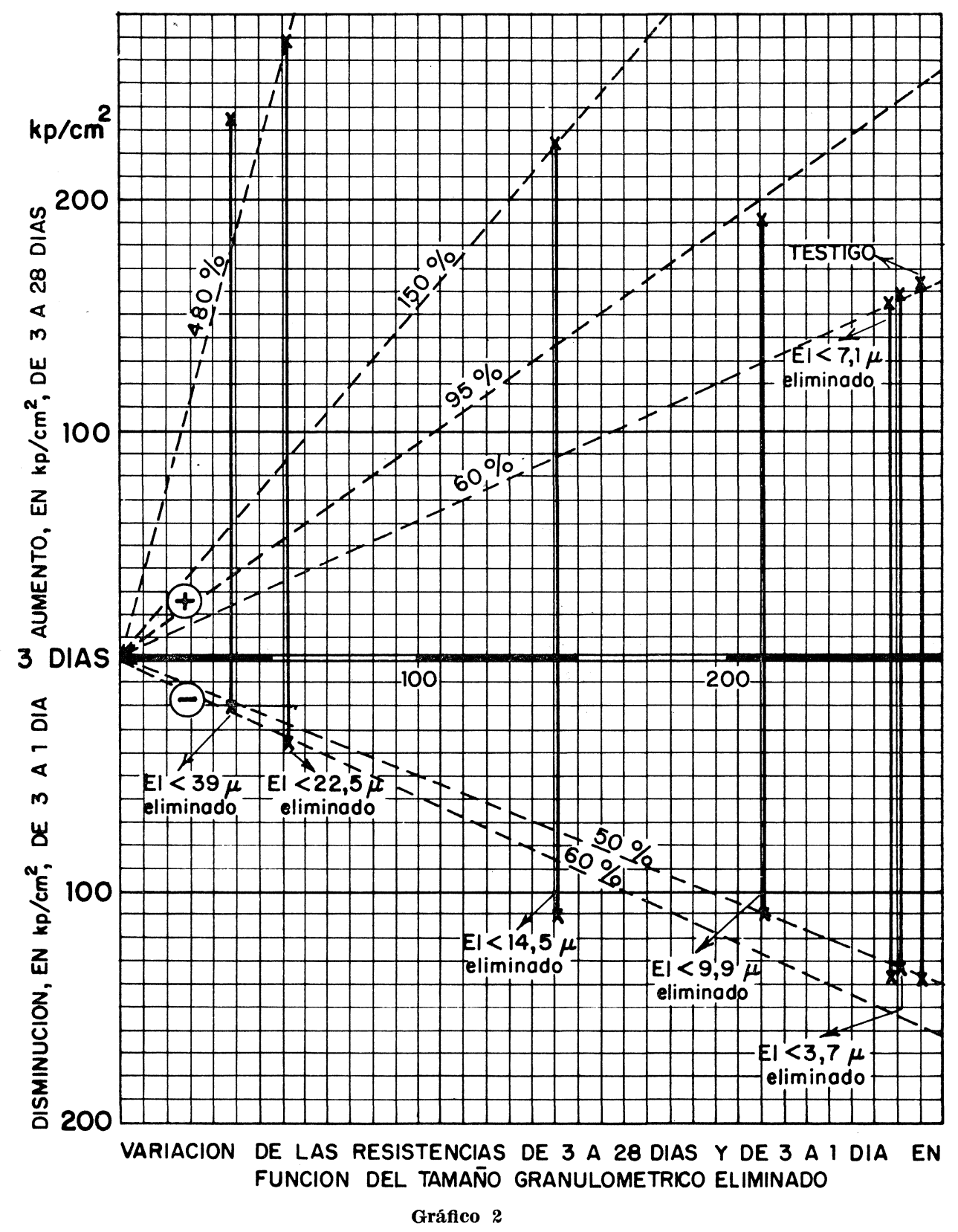

Después de ésto, creemos disponer de argumentos suficientemente válidos como para poder establecer las siguientes conclusiones:

1. En nuestro caso concreto, aparentemente, aunque quizás sea una excepción, no existen diferencias sensibles en la composición química de los distintos tamaños granulométricos. 
2. La superficie específica Blaine está sensiblemente afectada por la presencia de tamaños inferiores a 3,7 micras. Por lo tanto, además de otros motivos que no viene al caso enumerar, la superficie específica Blaine no se debe aceptar como representación válida y rigurosa de las resistencias.

3. Respecto a las resistencias debemos considerar los siguientes aspectos:

3.1. Los tamaños inferiores a 3,7 micras no intervienen de una forma patente en el desarrollo de las resistencias, tanto sean éstas de corto o largo plazo, más bien su presencia afecta de una forma sensible a la superficie Blaine.

3.2. A partir de las 9,9 micras, se observa que su principal influencia es en las resistencias a corto plazo, mientras que en las de 28 días no se detecta un efecto tan patente.

3.3. La eliminación correspondiente a 38 micras se deja sentir tanto se trate de corto como a largo plazo, aunque más bien su efecto es más sensible en las edades tempranas.

4. Según esto debemos resaltar el interés que encierra el fabricar un cemento cuya granulometría estuviera encuadrada entre los tamaños 3,7 a 50 micras (respecto a la limitación del tamaño menor no creo exista inconveniente para aceptarla).

5. En cambio, respecto a la de 50 micras puede existir alguna objeción y más aún cuando esta se ha deducido por extrapolación. Por lo tanto, y después de esta salvedad, cabe admitirse una mayor elasticidad o beligerancia y por ello, la limitación de 50 micras puede muy bien convertirse en 40 ó 60 micras.

6. Y como conclusión general podríamos establecer que, en la actualidad y debido a la forma en que se realiza la molienda, el cemento fabricado, una vez descontadas las adiciones inertes, se desaprovecha más del $30 \%$ de las posibilidades que de "per se" dispone el clínker en estado potencial. 